\title{
Anxiety level in context of chosen pro-health attitudes of male students during COVID-19 pandemic
}

\author{
Dorota Ortenburger ${ }^{1}$, Jacek Wąsik ${ }^{1, *}$, Iuliia Pavlova², Dariusz Mosler ${ }^{1}$ \\ ${ }^{1}$ Department of Kinesiology and Health Prevention, Jan Dlugosz University of Czestochowa, 42-200 Częstochowa, Poland \\ ${ }^{2}$ Theory and Methods of Physical Culture Department, Lviv State University of Physical Culture, 79007 Lviv, Ukraine
}

*Correspondence: j.wasik@ujd.edu.pl (Jacek Wąsik)

\begin{abstract}
Background and objective: Assessing the impact of different factors on anxiety level is a complex and challenging problem, especially during pandemic or similar life threatening situations. Stress can affect dietary and eating behaviors. The aim of this study is to extend knowledge concerning the relation between increased anxiety level during pandemic and attitude towards dietary and eating behaviors in the context of social situation and support of relatives and friends. This study was conducted in 2020 during COVID-19 pandemic.

Methods: State-Trait Anxiety Inventory alongside auxiliary questions about social relations and eating habits were asked to the male students from different universities and courses in Eastern Europe. To assess differences and dynamics of anxiety level, multiple regression models were used.

Results: Multiple regression models between state anxiety level with context to the following factors: paying particular attention to one's diet, namely, the way of nutrition during severe anxiety felt during the pandemic and factors such as strong social support and type of university course was $\mathrm{R}=0.41$ ( $p=$ $0.00)$. For trait-anxiety the same model returned $R=0.39(p=0.00)$. Analysis of variance revealed that support of relatives is significant factor for state anxiety level, whereas this factor is insignificant for trait anxiety level.

Conclusions: Models obtained from this study indicated that there are significant relations between anxiety level of male students and social support, which is expressed in the form of proper eating, therefore pro-health habits are revealed during elevated prolonged stress state such as COVID-19 pandemic.
\end{abstract}

\section{Keywords}

State anxiety; Trait anxiety; COVID-19; Social relations; Eating habits

\section{Introduction}

State of anxiety is a complex phenomenon, where gasping all factors that affects the state of a person are antagonized. There are many factors that affects anxiety level and decision making of individuals regarding their health is a state of uncertainty and feels under threat. Elevated anxiety levels are commonly related to external factors such as danger, threat, fear, and panic which are connected to actual situation that individual is confronting. State of anxiety warns person from chaos, entropy and loosing self-perception while confronting with natural threats [1].

Fear is a common emotion, which most humans experience during their lifetime. However, intensity and resistance to stress connected to fearful situation varies among individuals. Some people are more vulnerable to the feeling of 
anxiety, therefore it is assumed, that this phenomenon can be perceived as an individual trait of a person [2]. Anxiety is perceived to be an emotional state related to the processing of external or internal danger. This state is connected to increased stimuli in psychic, motor and autonomous system and fear-oriented mental activity [3-5].

Cognitive-based theories in psychology link vulnerability to abnormal stress level elevation along with tendency to shift attention and cognitive processing towards perceiving uncertain situations as dangerous ones [6]. This complexity divide anxiety to a state anxiety, which is understood as subjective, conscious awareness of being anxious and stressful with increased activity of autonomous nervous system. Traitanxiety is understood as personal behavioral predispositions, making individual prone to perceiving objectively neutral situation as threat and provoking elevated level of anxiety [7]. This concept of two forms of anxieties is connected to a current situation and external stimuli, whereas the other from of anxiety is connected to internal response to this stimuli, specific to individual traits of a person [8].

Elevated anxiety leads to increased stress level and development of somatic symptoms such as sleep disorders [9], increased muscle tension [10], prolonged state of being nervous, attention deficits [11], nausea, gastrointestinal and urological problems [12]. Eating disorders and poor dietary habits are often related to increased anxiety level not only among people with obesity [13], but also among healthy children, adolescents [14] and adults [15]. Proper diet has impact on the brain function. Limited vitamins and minerals intake can lead to development of anxiety and depression. Continuation of poor dietary habits while being in prolonged stress increases the chances of more severe symptoms related to anxiety disorders [16].

Pandemic of SARS-COV-2 virus started worldwide in 2020 which resulted in substantial changes in the lifestyle and risk perception of people because of prolonged lockdowns and restrictions as means of fighting this threat [17]. Fear and anxiety of COVID-19 causes psychological distress connected to career planning [18, 19] or spending leisure time [20]. People with preexisting anxiety-related disorders could worsen their condition during COVID-19 pandemic [21]. Health and economic anxiety surrounding COVID19 predict psychosocial distress because the sources of psychological distress associated with COVID-19 shifted away from early anxieties of physiological infection toward an emerging fear of joblessness stemming from the economic anxiety of the lockdown [22].

As quality of life declined, many researchers started to study state and trait anxiety among different populations. Researchers started to analyze different factors related to StateTrait Anxiety Inventory such as attitude [23], expecting a child [24, 25], infertility [26], cancer [27] or mental health of healthcare workers [28]. Eating disorders were well studied during pandemic, which resulted in systematic review by Miniati et al. [29].

In some cases, people experiencing stronger anxiety which is associated with the decrease in attention devoted to nutri- tion that is beneficial for the health [15]. Therefore, studies emphasized the role of cognitive and emotional factors in terms of making choices that relate to the type of food consumed. It is still unknown how different groups of people and their habits were affected by pandemic in context of their anxiety levels. The question arises as to whether the men, who during the ongoing COVID-19 pandemic are still in their course of studies, display differences in terms of the tendency to react with anxiety while undertaking protective action in the form of paying particular attention to pro-health behaviour. We hypothesize that there might be a relationship between the level of anxiety and factors related to nutrition and the sense of emotional support from close people. The hypothesis assumed that volatility of the anxiety level may be partly explained by these factors. The aim of our research is to expand knowledge on the subject of the relation between the level of intensity of the state anxiety felt and anxiety in the form of a certain personality predisposition and the attitude of paying particular attention to a diet during the course of the pandemic. These dependencies are additionally analysed by taking account of the feeling of differentiated degree of social support among students from their close ones.

\section{Methods}

\subsection{Participants}

The study includes 502 students from different universities on the courses related to social and physical culture studies. Detailed profiles of each study field are presented in Table 1.

\subsection{Measurement tools}

State-Trait Inventory (STAI) developed by Spielberger et al. [8] were use as primary questionnaire. State-Trait Anxiety Inventory is divided into two sections-State-Anxiety with 20 items and Trait-Anxiety, also with 20 items. First, the State Anxiety Scale (S-Anxiety) evaluates the current state of anxiety, asking how people feel "right now" and use items that measure subjective feelings of apprehension, tension, nervousness, worry, and activation of the autonomic nervous system. Second, the Trait Anxiety Scale (T-Anxiety) relates to predispositions (personal traits) and perceived anxiety. Responses to items on the S-Anxiety scale (to assess intensity of current feelings "at the present moment") [7]. In addition, authors created separate, additional supplementary questions in our research: 1. perception of support of close persons; 2. putting attention to diet while being anxious; 3 . worse nutrition during the pandemic than prior-to the occurrence of COVID-19. This was not a complete survey but only questions. The lower score indicated the lower level of the following variable.

\subsection{Statistical analysis}

Basic statistical analysis such as mean, standard deviation and variance analysis were performed. Regression analysis was used to study relation between state and trait anxiety levels with external factors (university, field of study) and internal 
T A B L E 1. Distribution of participants by study field and their age.

\begin{tabular}{lccc}
\hline Study field & Number of participants & Mean age & SD \\
\hline Military administration & 48 & 21.0 & 2.3 \\
State security & 143 & 21.7 & 2.5 \\
Law & 69 & 21.3 & 2.4 \\
Teacher-trainer (physical culture) & 41 & 20.2 & 1.0 \\
Physical therapist/occupational therapist & 44 & 19.8 & 1.3 \\
Hotel and restaurant business & 3 & 20.7 & 1.7 \\
Coach (sports) & 59 & 20.2 & 1.3 \\
Tourism & 4 & 19.3 & 0.4 \\
Graphic design & 1 & 18.0 & 0.0 \\
Foreign language teacher & 2 & 19.0 & 0.0 \\
Musical art & 2 & 19.5 & 0.5 \\
Fine arts. decorative arts. Restoration & 1 & 19.0 & 0.0 \\
Graphic design. Fine arts. decorative arts. Restoration & 3 & 20.0 & 0.0 \\
Performing arts & 5 & 20.0 & 1.7 \\
Translator & 2 & 19.0 & 0.0 \\
Physiotherapy & 43 & 20.7 & 1.9 \\
Physical education & 32 & 21.0 & 1.8 \\
\hline SD standard deviation. & & &
\end{tabular}

T A B L E 2. Multiple regression models of anxiety level in the context selected predictor variables.

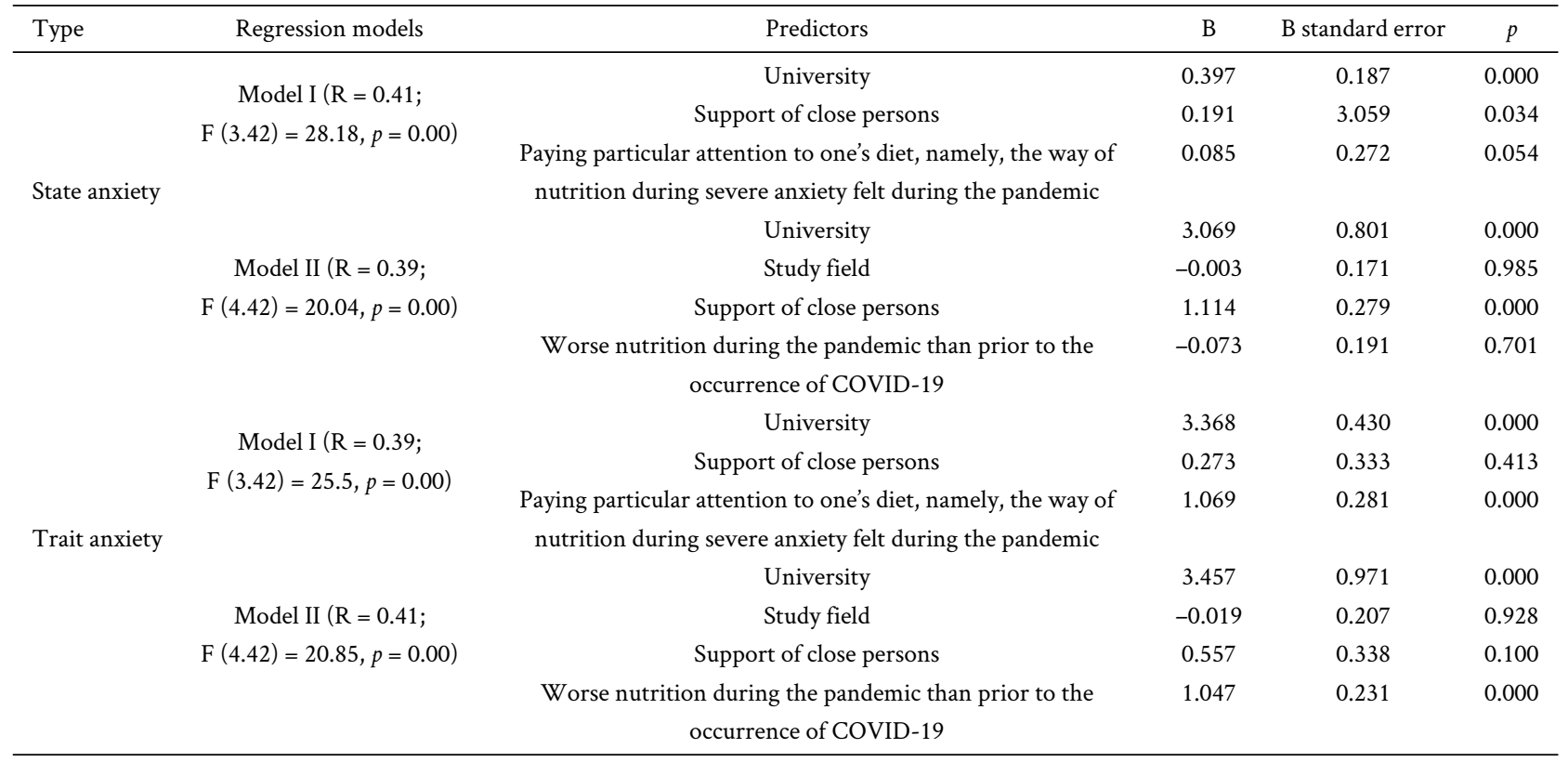

B, Unstandardized coefficient (obtained after running a regression model on variables measured in their original scales).

factors (support of close persons and focus on dietary habits during pandemics). The latter were expressed in multiple regression models. Analysis of variance (ANOVA) was used to explore relations between results of Sate-Trait Inventory results and scores from auxiliary questions.

\subsection{Ethics}

The study was conducted with the approval of Bioethics Committee of Lviv State University of Physical Culture with positive decision in protocol number 6 (2020-12-12). This study by questionnaire was conformed to the principles outlined in the Declaration of Helsinki. All participants were provided informed and signed consent before their inclusion in the study.

\section{Results}

Multiple regression analysis allowed to obtain two models for both state anxiety and trait anxiety separately (Table 2 ). For state anxiety, first model had higher $\mathrm{R}$ value $(\mathrm{R}=0.41)$ with only one significant predictor (university), while the second model $(\mathrm{R}=0.39)$ had two significant predictors (university and support of close persons). Trait anxiety model had reverse tendency. First model had $\mathrm{R}$ value which was lower than the second one $(R=0.39 ; R=0.41$ respectively). Both models had significant predictor of university and relation to eating habits by different predictors. First model predictor was related to paying attention to one's diet and the second was related to worse nutrition during pandemic.

Both state and trait anxiety levels of participant from 
T A B L E 3. Results of Stat-Trait Anxiety Inventory of participant from different universities.

\begin{tabular}{|c|c|c|c|c|c|c|}
\hline Type & University & Mean & $\mathrm{N}$ & $\mathrm{SD}$ & $\mathrm{F}$ & $p$ \\
\hline \multirow{4}{*}{ State anxiety } & National Academy of the National Guard of Ukraine & 48.85 & 260 & 4.58 & \multirow{4}{*}{23.09} & \multirow{4}{*}{0.000} \\
\hline & Lviv State University of Physical Culture, Ukraine & 51.75 & 151 & 4.00 & & \\
\hline & Ternopil Volodymyr Hnatiuk National Pedagogical University, Ukraine & 53.75 & 16 & 4.74 & & \\
\hline & Jan Dlugosz University, Poland & 47.75 & 75 & 4.78 & & \\
\hline \multirow{4}{*}{ Trait anxiety } & National Academy of the National Guard of Ukraine & 47.00 & 260 & 4.74 & \multirow{4}{*}{20.41} & \multirow{4}{*}{0.000} \\
\hline & Lviv State University of Physical Culture, Ukraine & 50.27 & 151 & 5.19 & & \\
\hline & Ternopil Volodymyr Hnatiuk National Pedagogical University, Ukraine & 53.62 & 16 & 6.54 & & \\
\hline & Jan Dlugosz University, Poland & 47.75 & 75 & 4.78 & & \\
\hline
\end{tabular}

N, number of participants; SD, standard deviation; F, Fisher's statistics.

T A B L E 4. Results of Stat-Trait Anxiety Inventory of participant from different study fields.

\begin{tabular}{|c|c|c|c|c|c|c|c|}
\hline & Field of study & $\mathrm{N}$ & Mean & Minimum & Maximum & $\mathrm{SD}$ & Statistical significance \\
\hline \multirow{7}{*}{ State anxiety } & physical education teacher & 79 & 50.24 & 40 & 62 & 4.45 & \multirow{7}{*}{$\mathrm{F}=5.582 ; p=0.000$} \\
\hline & state security & 143 & 49.22 & 38 & 59 & 3.86 & \\
\hline & military administration & 48 & 48.10 & 27 & 60 & 6.06 & \\
\hline & physiotherapy & 87 & 49.41 & 33 & 62 & 5.16 & \\
\hline & law & 69 & 48.64 & 20 & 57 & 4.75 & \\
\hline & coach & 59 & 52.17 & 45 & 63 & 3.64 & \\
\hline & others & 17 & 53.53 & 46 & 62 & 4.80 & \\
\hline \multirow{7}{*}{ Trait anxiety } & physical education teacher & 79 & 49.11 & 38 & 62 & 4.93 & \multirow{7}{*}{$\mathrm{F}=4.180 ; p=0.000$} \\
\hline & state security & 143 & 46.99 & 35 & 64 & 4.73 & \\
\hline & military administration & 48 & 47.25 & 38 & 66 & 5.27 & \\
\hline & physiotherapy & 87 & 48.89 & 33 & 62 & 5.67 & \\
\hline & law & 69 & 46.87 & 36 & 60 & 4.42 & \\
\hline & coach & 59 & 50.68 & 37 & 59 & 4.62 & \\
\hline & others & 17 & 53.35 & 40 & 69 & 6.60 & \\
\hline
\end{tabular}

$\mathrm{N}$, number of participants; SD, standard deviation.

different universities differs significantly. The highest scores were shown by participants from Ternopil Volodymyr Hnatiuk National Pedagogical University in Ukraine, while the lowest were among participants form Jan Dlugosz University in Poland (Table 3).

Both state and trait anxiety levels of participants from study fields were higher for the humanistic type of courses like tourism, music, and graphic arts (classified as others) with mean score exceeding 53 points. The lowest mean score in state anxiety level were shown in the group of military administration, while for trait anxiety level it was students of state security. The lowest noted score of individuals in the section of state anxiety belonged to the law student $(20$ points), while the highest note of 63 belonged to student of coaching course. The lowest noted trait anxiety score was much higher than that of state anxiety and belonged to student of physiotherapy with 33 points, and the highest noted value for all inventory belonged to a student of performing arts (classified in others) with 69 points. Overall, in the state anxiety measurements mean minimal to maximal scores ranges from 35.57 to 60.71 and from 36.71 to 63.14 for trait anxiety (Table 4).

Results obtained revealed that feeling of support of close friends or relatives is a significant factor in shaping scores of state anxiety level $(p<0.00041)$, while the same factor remains insignificant for scores of trait anxiety presented by participants $(p<0.723)$ (Fig. 1). Both types of diet habits reports exhibited significant impact on both types of anxiety levels. Reports of bad diet behaviors (lower scores) were associated with the higher values of state anxiety, but tendency became leveled as individuals had higher opinion about their bad habits. On the other hand, the higher points participants reported for bad habits, higher were their points for trait anxiety (Fig. 2).

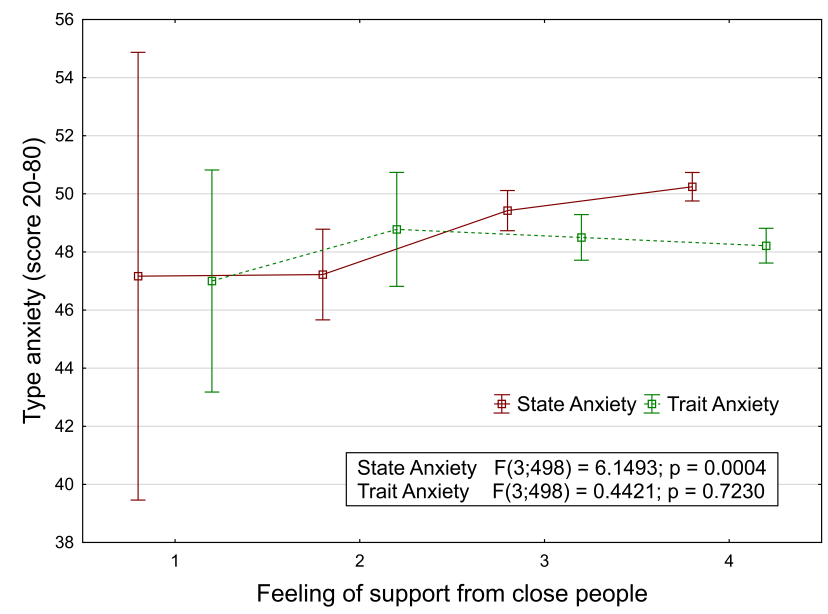

F I G. 1. The level of anxiety of participants and feeling support from close them people.

Participants placed higher scores for putting attention to 


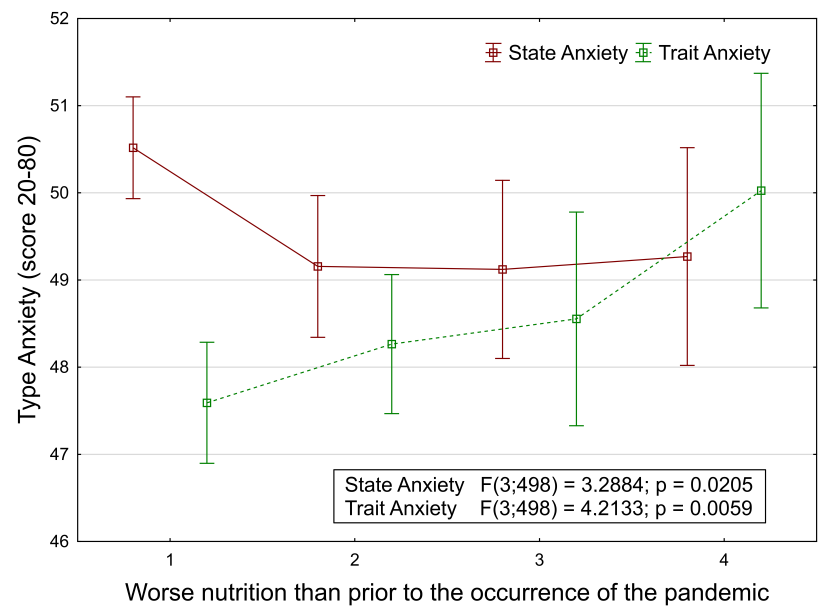

F I G. 2. Anxiety level (state and trait anxiety results) and subjective assessment sense of worse nutrition during the pandemic than prior to the occurrence of COVID-19.

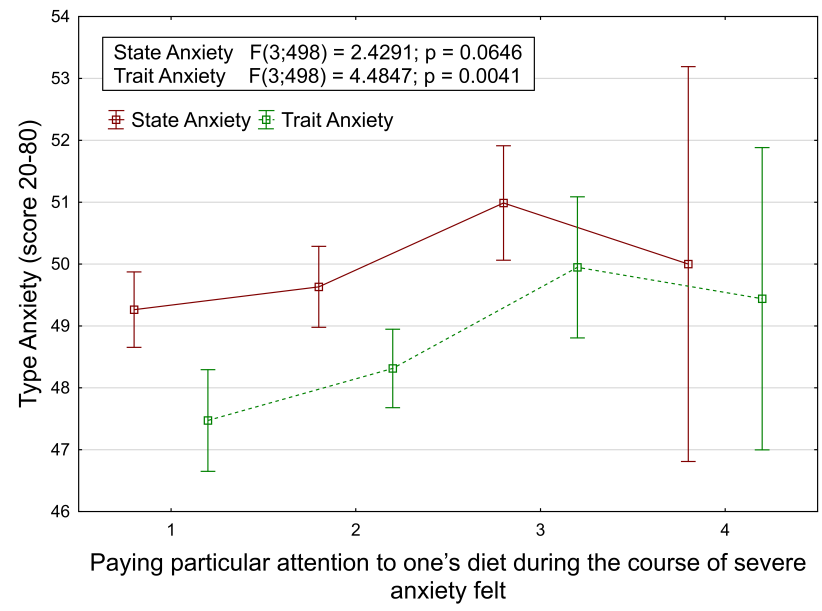

F I G. 3. Paying particular attention to one's diet while severe anxiety felt while the pandemic.

their diet alongside higher points for state and trait anxiety from 1 to 3 points, then mean score of both state and trait anxiety were lower for maximum value that could be granted with the highest range of scores from minimal to maximal values. For both types of anxiety, impact of reported attention for diet habits was significant (Fig. 3).

\section{Discussion}

Studies of anxiety level during pandemic is justified by the fact, that acute stress is transformed into chronic one by cognitive factor. Being worried repeatedly and anticipation of negative outcomes of current situation is a result of persistent stress caused by ongoing life-threating factors [30]. This mechanism has biological, atavistic basis of survival instincts. According to a game theory, it is better to assume, that unknown threat is life-threatening and avoid danger rather that curiosity and risk of being killed [31].

Following are the key preliminary findings that emerged from our analysis. The data obtained in our study supports the existence of connection between STAI results and factors such as university and study field. The result of this study varies from those obtained by other authors from different regions of the world. Turkish students reveals state anxiety level ranging from 42 to 46.3 points [32], which is lower than the participants of this study. Similarly nursing students from Spain showed anxiety levels which ranged from 50 to 58 points [33], which was concurrent with presented results. Similar results were also obtained from the students in China [34]. Most of the published reviews are limited to prevalence results of anxiety [35-37] and its own regression models, without presenting raw STAI results. However, all mentioned study uses the same auxiliary questions regarding their analysis factors in the Likert scale, as presented in our study.

Significant relation for factors such as paying attention to one's diet during sever anxiety felt during pandemic and factors such as strong social support and type of university courses being taken are proven by value of coefficient of determination for both models $\left(\mathrm{R}^{2}=0.17 ; \mathrm{R}^{2}=0.16\right)$ for state anxiety $(p<0.000)$ and respectively $\mathrm{R}^{2}=0.15 ; \mathrm{R}^{2}=$ 0.16 for trait anxiety $p<0.000$. Our results indicate, that coefficient values, which determines degree of regression of the data are not high, but its significance could be interpret within the scope of the presented models. The clarification of the relationship between the intensity of anxiety and the dependent variables (predictors) required the application of the least square method of the evaluation of parameters. The precision of the adjustment of the model to the data was evaluated by means of the coefficient of multiple determination of R. A statistical model was obtained which showed a slight, yet noticeable systematic trend in the data leaving aside the random variability.

In our current study eating behavior is presented as internal factor. As it was proven to be significant in terms of participants anxiety level. We could refer this phenomenon to informational metabolism, which is considered to be a conception that extends metabolism from purely chemical transformation of eaten compounds to emotional-rational experience, with exchange of emotions while consuming food [38]. Emotions play a crucial role in decision making as rational and cognitive processing of current problems are affected by cognitive bias of strong emotions, affecting our judgment [39]. Our results of auxiliary question regarding eating habits is biased for personal perception of one's actions. In comparison, this is not the same result as actual study on an individual diet of a person, but is rather self-awareness about necessity of paying attention to eating habits. This could be perceived as sedative agents for emotional control over own habits. Belief in eating healthy, therefore taking care of own body could help regain control during elevated anxiety period, which helps control sleep and rest time [40]. High stress level and inability to control health-related habits could be the main factor contributing towards developing additional somatic health problems. When using the dosed physical activity in programs of physical rehabilitation, many patients have been shown to improve their functional state, 
physical performance, quality of life, promote their mental stability, and gives them confidence in the future [16, 41, 42].

Emotional support of acquaintances such as life partner, family or friends is also significant contributing factor for stress management and state anxiety level. Although sense of being supported was categorized as internal factor but objectively interpreting, the presence of support is external, as it is given by others. During pandemic people face two scenarios involving lockdown-loneliness and social distancing from close people [43] or being locked with people in the small area for prolonged period of time, which could lead to increased tension between partners and can even lead to violence [44].

First, although questionnaire data are widely recognized to be reliable and valid [8], it does not necessarily provide unbiased population estimates. Future studies should consider employing more research tools regarding both anxiety measurement and dietary habits tools. As this study did not cover adequate social context data, we could assume that either people with high sense of emotional support have good long-distance online contact with friends and family or they have good relations with people they are living with. Putting this context with eating habits, we cannot exclude possibility that students eating habits were managed by family members or partners and their attention toward eating healthy were limited to perception of events such as healthy meal. Presented reasoning could serve as limitation of this study, where exploratory factors need to be known to obtain knowledge about the reasons of this study results.

Worse nutrition during the pandemic compared to nutrition prior to the occurrence of COVID-19 can be deleterious to the mental health of men. These findings suggest that health-related spending on COVID-19 must include significant allocations of funds for developing knowledge emphasizing the influence of diet on mental health. For example, internet-based cognitive behavioral therapy can play an important role in improving eating habits.

Specificity of this study lies in the region where participants live. Besides pandemic, there is ongoing military conflict in Ukraine. It was proved that threat of conscription to army of students could affect their anxiety levels [45]. Therefore, pandemic cannot be perceived as single factor elevating anxiety level in this study. However, group of participants from military-related field of study did not reveal any significant higher level of anxiety compared to peers from other fields; therefore, it could be stated that political factors were dimmed by the threat of pandemic.

\section{Conclusions}

Conducted study indicates that there is relationship between the variables of confirmed significance which substantiates the fact that the feeling of social support and paying particular attention to nutrition during the pandemic (treated as a sign of pro-health behavior) may in varying degrees be associated with the level of anxiety. Our current study shows that type of university and field of study are significant factors of anxiety level among students and students at pedagogical university type and humanistic type of studies showed higher scores of STAI than students from courses in the field of physical culture.

Sense of emotional support from partners, friends and relatives could be perceived as significant external factor in determining the state anxiety level as opposed to the initial assumption of being internal factor. Focusing attention on the quality of diet is related to trait anxiety, indicating that personal traits and characteristic of individual attitude toward health could probably be related to overall stress management capacities of a person, rather than response to pandemic based on COVID-19 situation.

\section{Abbreviations}

AAA, abdominal aortic aneurysm; AVF, arteriovenous fistula; IVC, inferior vena cava; CRRT, continuous renal replacement therapy; CT, computed tomography; ICU, intensive care unit; ALT, alanine transaminase; AST, aspartate transaminase; TBIL, total bilirubin; DBIL, direct bilirubin.

\section{Author contributions}

DO and JW conceived and designed the experiments. DO, IP, JW, DM performed the experiments. IP and JW Data Curation. DO, JW, DM analyzed the data. DO, JW, DM and IP wrote the paper. JW Supervision, Project Administration and Funding Acquisition. All authors have read and agreed to the published version of the manuscript.

\section{Ethics approval and consent to participate}

Informed consent was obtained from all subjects involved in the study. The study regarding analysis of wellbeing during the COVID-19 pandemic is accepted by approval of Bioethics Committee of Lviv State University of Physical Culture with protocol number 6 (2020-16-12).

\section{Acknowledgment}

Thanks to all the peer reviewers for their opinions and suggestions.

\section{Funding}

This research received no external funding.

\section{Conflict of interest}

The authors declare no conflict of interest.

\section{References}

[1] Ceklarz J. Revision of the Conception of Antoni Kępiński’s Information Metabolism. Psychiatria Polska. 2018; 52: 165-173.

[2] Endler NS, Kocovski NL. State and trait anxiety revisited. Journal of Anxiety Disorders. 2001; 15: 231-245.

[3] Pacesova P, Smela P, Kracek S. Personal well-being as part of the quality of life: Is there a difference in the personal well-being of women and men with higher level of anxiety trait regarding their sport activity? Physical Activity Review. 2019; 7: 201-208. 
4] Reinholdt-Dunne ML, Mogg K, Bradley BP. Attention control: relationships between self-report and behavioural measures, and symptoms of anxiety and depression. Cognition and Emotion. 2013; 27: 430-440.

[5] Proios I, Batsiou S, Bebetsos E, Malliou P, Fotiadou E, Proios M. Achievement goal orientations profile in people with physical disability. Physical Activity Review. 2019; 7: 9-17.

[6] Blakey SM, Abramowitz JS. The effects of safety behaviors during exposure therapy for anxiety: Critical analysis from an inhibitory learning perspective. Clinical Psychology Review. 2016; 49: 1-15.

[7] Spielberger CD. State-Trait Anxiety Inventory. 2010. Available at: https://doi.org/10.1002/9780470479216.corpsy0943 (Accessed: 5 May 2021)

[8] Spielberger C, Gorsuch R, Lushene R, Vagg PR, Jacobs G. Manual for the State-Trait Anxiety Inventory (Form Y1-Y2). CA: Consulting Psychologists Press. 1983.

[9] Taylor DJ, Lichstein KL, Durrence HH, Reidel BW, Bush AJ. Epidemiology of insomnia, depression, and anxiety. Sleep. 2005; 28: 1457-1464.

[10] Pluess M, Conrad A, Wilhelm FH. Muscle tension in generalized anxiety disorder: a critical review of the literature. Journal of Anxiety Disorders. 2009; 23: 1-11.

[11] Pacheco-Unguetti AP, Acosta A, Callejas A, Lupiáñez J. Attention and Anxiety: Different Attentional Functioning Under State and Trait Anxiety. Psychological Science. 2010; 21: 298-304.

[12] Mussell M, Kroenke K, Spitzer RL, Williams JBW, Herzog W, Löwe B. Gastrointestinal symptoms in primary care: prevalence and association with depression and anxiety. Journal of Psychosomatic Research. 2008; 64: 605-612.

[13] Schulz S, Laessle RG. Associations of negative affect and eating behaviour in obese women with and without binge eating disorder. Eating and Weight Disorders. 2010; 15: e287-e293.

[14] Kim JY, Kang HL, Kim D, Kang SW, Park YK. Eating Habits and Food Additive Intakes are Associated with Emotional States Based on EEG and HRV in Healthy Korean Children and Adolescents. Journal of the American College of Nutrition. 2017; 36: 335-341.

[15] Yannakoulia M, Panagiotakos DB, Pitsavos C, Tsetsekou E, Fappa E, Papageorgiou $\mathrm{C}$, et al. Eating habits in relations to anxiety symptoms among apparently healthy adults. a pattern analysis from the ATTICA Study. Appetite. 2008; 51: 519-525.

[16] Melanson KJ. Nutrition Review: Relationships of Nutrition with Depression and Anxiety. American Journal of Lifestyle Medicine. 2007; 1: 171-174.

[17] Bottemanne H, Morlaàs O, Fossati P, Schmidt L. Does the Coronavirus Epidemic Take Advantage of Human Optimism Bias? Frontiers in Psychology. 2020; 11: 2001.

[18] Mahmud MS, Talukder MU, Rahman SM. Does 'Fear of COVID-19' trigger future career anxiety? An empirical investigation considering depression from COVID-19 as a mediator. International Journal of Social Psychiatry. 2020; 67: 35-45.

[19] Mahmud MS, Rahman MM, Masud-Ul-Hasan M, Islam MA. Does 'COVID-19 phobia' stimulate career anxiety?: Experience from a developing country. Heliyon. 2021; 7: e06346.

[20] Servidio R, Bartolo MG, Palermiti AL, Costabile A. Fear of COVID19 , depression, anxiety, and their association with Internet addiction disorder in a sample of Italian students. Journal of Affective Disorders Reports. 2021; 4: 100097.

[21] Shafran R, Rachman S, Whittal M, Radomsky A, Coughtrey A. Fear and Anxiety in COVID-19: Preexisting Anxiety Disorders. Cognitive and Behavioral Practice. 2021. (in press)

[22] Timming AR, French MT, Mortensen K. Health anxiety versus economic anxiety surrounding COVID-19:an analysis of psychological distress in the early stages of the pandemic. Journal of Affective Disorders Reports. 2021; 5: 100152

[23] Lin Y, Hu Z, Alias H, Wong LP. Knowledge, Attitudes, Impact, and Anxiety Regarding COVID-19 Infection Among the Public in China. Frontiers in Public Health. 2020; 8: 236.

[24] Davenport MH, Meyer S, Meah VL, Strynadka MC, Khurana R. Moms
Are Not OK: COVID-19 and Maternal Mental Health. Frontiers in Global Women's Health. 2020; 1: 1-6.

[25] Hessami K, Romanelli C, Chiurazzi M, Cozzolino M. COVID-19 pandemic and maternal mental health: a systematic review and metaanalysis. Journal of Maternal-Fetal and Neonatal Medicine. 2020; 1-8. (in press)

[26] Esposito V, Rania E, Lico D, Pedri S, Fiorenza A, Strati MF, et al. Influence of COVID-19 pandemic on the psychological status of infertile couples. European Journal of Obstetrics \& Gynecology and Reproductive Biology. 2020; 253: 148-153.

[27] Nardone V, Reginelli A, Vinciguerra C, Correale P, Calvanese MG, Falivene S, et al. Mood Disorder in Cancer Patients Undergoing Radiotherapy During the COVID-19 Outbreak. Frontiers in Psychology. 2021; $12: 568839$.

[28] Di Tella M, Romeo A, Benfante A, Castelli L. Mental health of healthcare workers during the COVID-19 pandemic in Italy. Journal of Evaluation in Clinical Practice. 2020; 26: 1583-1587.

[29] Miniati M, Marzetti F, Palagini L, Marazziti D, Orrù G, Conversano C, et al. Eating Disorders Spectrum during COVID Pandemic: a systematic review. MedRxiv. 2021. (in press)

[30] Loades ME, Chatburn E, Higson-Sweeney N, Reynolds S, Shafran R, Brigden A, et al. Rapid Systematic Review: the Impact of Social Isolation and Loneliness on the Mental Health of Children and Adolescents in the Context of COVID-19. Journal of the American Academy of Child \& Adolescent Psychiatry. 2020; 59: 1218-1239. e3.

[31] Ma Z. Towards an Extended Evolutionary Game Theory with Survival Analysis and Agreement Algorithms for Modeling Uncertainty, Vulnerability, and Deception. Berlin, Heidelberg: Springer. 2009.

[32] Ayran G, Köse S, Küçükoğlu S, Aytekin Özdemir A. The effect of anxiety on nicotine dependence among university students during the COVID-19 pandemic. Perspectives in Psychiatric Care. 2021. (in press)

[33] García-González J, Ruqiong W, Alarcon-Rodriguez R, RequenaMullor M, Ding C, Ventura-Miranda MI. Analysis of Anxiety Levels of Nursing Students Because of e-Learning during the COVID-19 Pandemic. Healthcare. 2021; 9: 252.

[34] Yang H, Chen Z, Fan Y, Hu X, Wu T, Kang S, et al. Knowledge, attitudes and anxiety toward COVID-19 among domestic and overseas Chinese college students. Journal of Public Health. 2021; fdaa268. (in press)

[35] Lasheras I, Gracia-García P, Lipnicki DM, Bueno-Notivol J, LópezAntón R, de la Cámara C, et al. Prevalence of Anxiety in Medical Students during the COVID-19 Pandemic: A Rapid Systematic Review with Meta-Analysis. International Journal of Environmental Research and Public Health. 2020; 17: 6603.

36] Dyrbye LN, Thomas MR, Shanafelt TD. Systematic review of depression, anxiety, and other indicators of psychological distress among U.S. and Canadian medical students. Academic Medicine. 2006; 81: 354-373

37] Jahrami H, BaHammam AS, Bragazzi NL, Saif Z, Faris M, Vitiello MV. Sleep problems during the COVID-19 pandemic by population: a systematic review and meta-analysis. Journal of Clinical Sleep Medicine. 2021; 17: 299-313.

[38] Bielecki A, Nowak D. A Multi-agent System Based on the Information Metabolism Theory. Parallel Processing and Applied Mathematics. 2004; 72: 439-446.

[39] Britton G, Bailey H. Attention bias modification effects on interpretive bias for fear of positive and negative evaluation in social anxiety. Clinical Neuropsychiatry Journal of Treatment Evaluation. 2018; 15: 94-104.

[40] Geiker NRW, Astrup A, Hjorth MF, Sjödin A, Pijls L, Markus CR. Does stress influence sleep patterns, food intake, weight gain, abdominal obesity and weight loss interventions and vice versa? Obesity Reviews. 2018; 19: 81-97.

[41] Steimer T. The biology of fear- and anxiety-related behaviors. Dialogues in Clinical Neuroscience. 2002; 4: 231-249.

[42] Kerr-Gaffney J, Harrison A, Tchanturia K. Social anxiety in the eating disorders: a systematic review and meta-analysis. Psychological Medicine. 2018; 48: 2477-2491. 
[43] Cauberghe V, Van Wesenbeeck I, De Jans S, Hudders L, Ponnet K. How Adolescents Use Social Media to Cope with Feelings of Loneliness and Anxiety during COVID-19 Lockdown. Cyberpsychology, Behavior, and Social Networking. 2021; 24: 250-257.

[44] Every-Palmer S, Jenkins M, Gendall P, Hoek J, Beaglehole B, Bell C, et al. Psychological distress, anxiety, family violence, suicidality, and wellbeing in New Zealand during the COVID-19 lockdown: A crosssectional study. PLoS ONE. 2020; 15: e0241658.
[45] Pavlova I, Zikrach D, Mosler D, Ortenburger D, Gora T, Wasik J. Determinants of anxiety levels among young males in a threat of experiencing military conflict-Applying a machine-learning algorithm in a psychosociological study. PLoS ONE. 2020; 15: e0239749. 